\title{
Motivation and Evaluation in Education from the Sustainability Perspective: A Review of the Scientific Literature
}

\author{
José Sánchez-Santamaría $\left.{ }^{1} * \mathbb{(}\right)$, Brenda Imelda Boroel-Cervantes ${ }^{2}\left(\mathbb{D}\right.$, Fátima-María López-Garrido ${ }^{1}$ \\ and David Hortigüela-Alcalá ${ }^{3}$ (D) \\ 1 Department of Pedagogy, Universidad de Castilla-La Mancha, 16071 Cuenca, Spain; \\ fatimamaria.lopez1@alu.uclm.es \\ 2 Faculty-of-Administrative-and-Social-Sciences, University Autonomous of Baja California, Ensenada 22860, Mexico; \\ brenda.boroel@uabc.edu.mx \\ 3 Department of Specific Didactics, Faculty of Education, University of Burgos, 09001 Burgos, Spain; \\ dhortiguela@ubu.es \\ * Correspondence: jose.ssantamaria@uclm.es; Tel.: +34-926-295-300
}

\section{check for}

updates

Citation: Sánchez-Santamaría, J.; Boroel-Cervantes, B.I.; López-Garrido, F.-M.; Hortigüela-Alcalá, D.

Motivation and Evaluation in Education from the Sustainability Perspective: A Review of the Scientific Literature. Sustainability 2021, 13, 4047. https://doi.org/ $10.3390 /$ su13074047

Academic Editors: Dina Belluigi and Vasiliki Brinia

Received: 31 January 2021

Accepted: 29 March 2021

Published: 6 April 2021

Publisher's Note: MDPI stays neutral with regard to jurisdictional claims in published maps and institutional affiliations.

Copyright: (C) 2021 by the authors. Licensee MDPI, Basel, Switzerland. This article is an open access article distributed under the terms and conditions of the Creative Commons Attribution (CC BY) license (https:// creativecommons.org/licenses/by/ $4.0 /)$.

\begin{abstract}
Background: This paper outlines the results of a literature review of meta-analyses published on motivation and evaluation in the last five years. (2) Methods: A systematic review of three educational databases (WoS, SCOPUS and ERIC) was conducted following the PRISMA and PICO approaches. A total of 54 peer-reviewed meta-analysis papers were selected, analysed and compared. (3) Results: A significant number and variety of meta-analyses have been conducted: motivation meta-analyses focus primarily on contextual variables, self-regulation and students' academic performance, and evaluation meta-analyses examine the effectiveness of the teaching intervention, the use of teaching methodologies and technological resources for learning. (4) Conclusions: There are two important absences: on the one hand, it is necessary to develop meta-analyses that combine motivation and evaluation, also measuring their interaction, from the perspective of sustainability, and not only of educational improvement, and on the other hand, it is necessary to perform meta-analyses on the effectiveness of the formative and shared evaluation of the sustainability of learning processes.
\end{abstract}

Keywords: motivation; evaluation; learning; meta-analysis; sustainability

\section{Introduction}

A quality education inspired by the social value of sustainability can be established as one that is able to provide meaningful and relevant learning environments, processes and tools for all students, ensuring access, promoting retention and contributing to the educational success of all in alignment with SDG number 4 of the 2030 Agenda framework: guaranteeing an inclusive, fair and quality education, and promoting opportunities for lifelong learning for all [1]. Furthermore, this kind of education must also promote the acquisition of reflective and critical competences for the development of a citizenship committed to the current challenges from a transversal, holistic and complex perspective $[2,3]$. Hargreaves and Fink [4] refer to such a scenario as sustainable education systems or actions to raise learning levels for all students, reduce learning gaps and increase public commitment to financing education. Therefore, we must consider an education inspired by sustainability as a framework for ensuring experiences that can positively impact on the development of a whole person's capabilities, including lifelong learning from the capabilities perspective. Under the premise of sustainable education, shared learning spaces are to be created, where different agents can promote the development of the learner's competences. This means that educational actors can be aware of their capacity to learn, to generate change and to make policy decisions through discourses that articulate and sustain good practices, as well as innovative approaches to new educational demands. 
As stated above, this approach is related to the requirement of quality education. It should make it possible to change the aspects that can be improved and maintain those that work. All of this is based on criteria of quality and equity to guarantee successful learning experiences for all. In this sense, some dilemmas arise without a simple answer. However, this highlights the importance of spaces for intersubjective construction and collaboration in order to move towards quality education for all. Thus, an education inspired by the principle of sustainability must be able to create contexts, resources and processes for all learners to succeed [5].

Three questions are central to the deployment of successful education for all from the perspective of sustainability: (1) Contexts for successful learning must favour organisational, methodological and cultural conditions connected to the pedagogical principles of any learning action. (2) Resources are useful materials and instruments at the service of learning and teaching. Every resource has the role of helping the teacher to fulfil his or her educational function and the student to learn in coherence with the needs and objectives of the teaching-learning process. (3) The design and development of effective educational processes is essential for successful learning. Within this aspect, two processes are essential: The first educational process is linked to the motivation that allows the learners themselves to be guided towards well-defined learning outcomes [6]. The second educational process refers to assessment as a learning activity that enables learners to become aware of what and how they learn as acts of metacognition and self-regulation [7-9]. Both educational elements should not be interpreted as isolated elements but as processes aimed at enabling everyone to be producers and executors in self-academic, formative and professional itineraries sustained in their life projects in coexistence with others.

The aim of this paper is to map published meta-analyses on motivation and assessment throughout the teaching-learning process. To this end, a systematic review was carried out to serve as an umbrella and closure of the sustainability monograph in which this proposal is included.

\section{Materials and Methods}

A systematic review brings a focused view of a relevant issue of interest for the development of educational research $[10,11]$. It is thus a methodological strategy to screen, evaluate, synthesise and analyse the existing literature on the topic of motivation and evaluation or assessment in education.

\subsection{Search Sources}

This systematic review focuses on articles published in the last 5 years (January 2016March 2021); concretely, meta-analyses included in the WoS, SCOPUS and ERIC databases are analysed. The search strategy was based on the use of terms on the subject of the study. The descriptors used were: motivation, evaluation, assessment and education. The search operators AND and OR were used.

\subsection{Criteria for Inclusion and Exclusion of Articles}

Inclusion criteria were as follows: (1) meta-analysis studies; (2) English language, (3) meta-analyses referring to motivation, evaluation and assessment; (4) articles collected from WoS, Scopus and ERIC databases; (5) articles within the field of educational research.

Exclusion criteria were as follows: (1) duplicate articles, (2) articles with limited information on content and methodology and (3) articles not within the time period of our analysis (January 2016 to March 2021).

\subsection{Limits and Methodology of the Search}

The search procedure was based on the application of the Preferred Reporting Items for Systematic Reviews and Meta-Analyses (PRISMA) [10]. The PICO strategy was also used to find key data on the following: year, country, studies, sample or participants, purpose, content and outcomes. 
This review has at least four limitations to be taken into account, since it does not seek representativeness but rather the relevance and significance of the published works: (1) it includes studies published in the last five years; (2) the selection of articles is generic and not based on their internal content; (3) it assumes a broad sense of sustainability; (4) it only incorporates meta-analyses, ignoring articles that are systematic reviews.

\subsection{Procedure}

The time period for the identification and selection of articles based on meta-analyses on the research topics was between 1 December 2020 and 20 March 2021.

The stages of the research were as follows: (1) Setting criteria for inclusion/exclusion of articles. (2) Decision making on the databases to be reviewed for information extraction. Three databases were selected: WoS, Scopus and ERIC. (3) Defining the search descriptors, which was among the difficulties of the study. After an exhaustive review, it was decided to use three descriptors: motivation, evaluation and assessment. All three descriptors are widely accepted by the scientific community, and are used unambiguously and commonly in the literature. The problem was that there are a large number of papers published on these topics every year, which made the research inoperative. Even the shorter time period than the one finally chosen yielded a large quantity of publications. This suggested the possibility of separate searches but also the need to focus on a particular type of study. The results of separate searches were, on the one hand, motivation articles, and on the other hand, evaluation/assessment articles.

The search strategy combined two steps for identifying and selecting information: the first step, motivation AND meta-analysis, evaluation OR assessment AND meta-analysis, and the second step, motivation AND meta-analysis (educational research), evaluation OR assessment AND meta-analysis (educational research). (5) The articles were selected using the analysis tools of the different databases, and then incorporated into MENDELEY ${ }^{\circledR}$ to be read, analysed and compared. This process involved all the authors of this paper following an interjudge agreement procedure to make decisions on the suitability, relevance and fit of the selected articles. The result was a realistic and useful number of articles that were considered for further analysis.

The search strategy was systematised using the PRISMA Flow Diagram template [10]. Its usefulness lies in its visual power and coherent organisation of the flow of action with its decisions and results. Thus, for each of the descriptors, motivation and evaluation/assessment, the initial search yielded a total of 98 documents. These documents were refined according to the criteria. After eliminating duplicates and a primary analysis (title, abstract and keywords), 69 records were explored by applying the inclusion/exclusion criteria to refine the selection through a detailed reading by the researchers, individually, and then shared, of the documents suitable for study according to the objectives of this research (Figure 1).

Six categories of analysis were used:

(1) Author and year of publication: Which authors are involved in the meta-analysis? In which year was it published? The search period was the last five years in order to obtain a sufficient but manageable information base.

(2) Country: descriptive but illustrative of where this type of study is produced and prioritized.

(3) Studies: essential questions in meta-analyses. The studies used to carry out the systematic review provide information on the quality of the meta-analysis and its implications.

(4) Sample: as with the previous criterion, in a meta-analysis, it is necessary to identify the sample used, not always the number of people, but the number of studies.

(5) Purpose: the objective of meta-analysis.

(6) Content: thematic descriptors that allow concepts to be associated with the descriptors of our study; it is essential to use the Boolean data search in English and Spanish. Therefore, each data set of the two levels refers to the established search descriptors. 


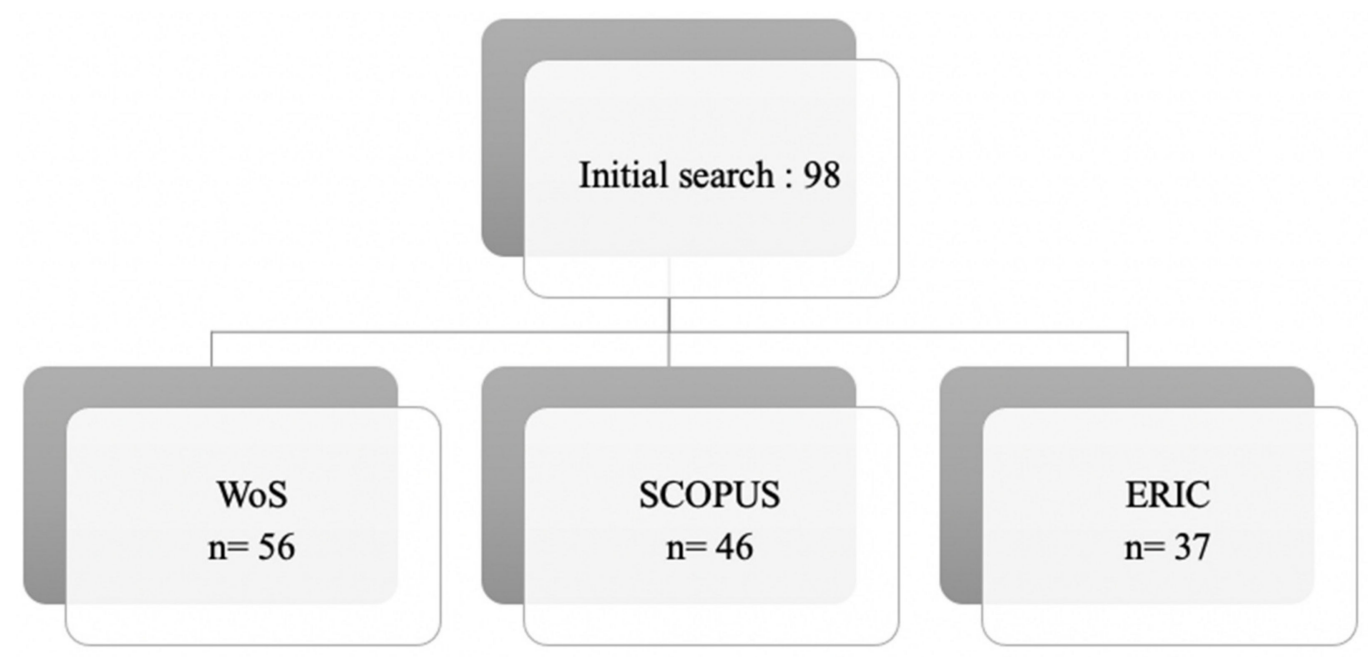

First level of exclusion:

- Duplicated articles

- Not published in journals indexed in JCR or SCOPUS

- In other languages than English or Spanish:

Potential relevant studies $n=67$

Second Level of exclusion:

- Information-poor articles

- Articles with methodological limitations

Final included studies $n=54$

Figure 1. Flow diagram of the systematic search process.

\subsection{Quality Assessment}

The quality of the selection process, as well as the adequate implementation of the inclusion and exclusion criteria, was carried out by combining four strategies: (1) Inclusion of the review in PROSPERO of the Centre for Review and Dissemination. Application 
of the PRISMA protocol [10]. This was also combined with the use of AMSTAR [11], an excellent tool of critical appraisal for systematic reviews. (3) The Consolidated Standards of Reporting Trials Statement [12] was used. It is a set of 25 recommendations to inform trial design, analysis and interpretation. The inherent nature of meta-analyses recommended that this strategy also be applied. (4) The search procedure was initially conducted anonymously by two investigators, and the results were discussed by interjudge agreement. In the case of doubt, the register was consulted again, and collegial decisions were made.

\section{Results}

The results are a descriptive and comparative analysis of 53 meta-analyses.

\subsection{Descriptive Analysis}

The descriptive analysis shows the main key indicators of the sample of publications selected in this study. For this purpose, a table format was chosen to summarise the descriptive information for each of them. Information on the descriptor motivation is shown in Table 1, and information on the evaluation and assessment is shown in Table 2.

\subsection{Visual Comparative Analysis: Key Words}

The comparative analysis informs five issues: (1) In recent years, we have witnessed a proliferation in the use of meta-analysis to provide empirical evidence on the effects of variables related to motivation, evaluation and assessment in education. (2) There is an important variety of issues concerning the topic of motivation and evaluation, although (3) motivation and assessment are jointly addressed when meta-analyses deal with the topics of testing, achievement or learning improvement. (4) Meta-analyses on motivation are mainly focused on the context and educational process variables, while meta-analyses on assessment are focused on the effectiveness of the intervention, the use of methodologies or technological resources or self-regulation in learning. (5) It is remarkable that there are very few meta-analyses on formative and shared assessment.

Figures 2 and 3 show the key descriptors related to the meta-analysis studies analysed in this article.
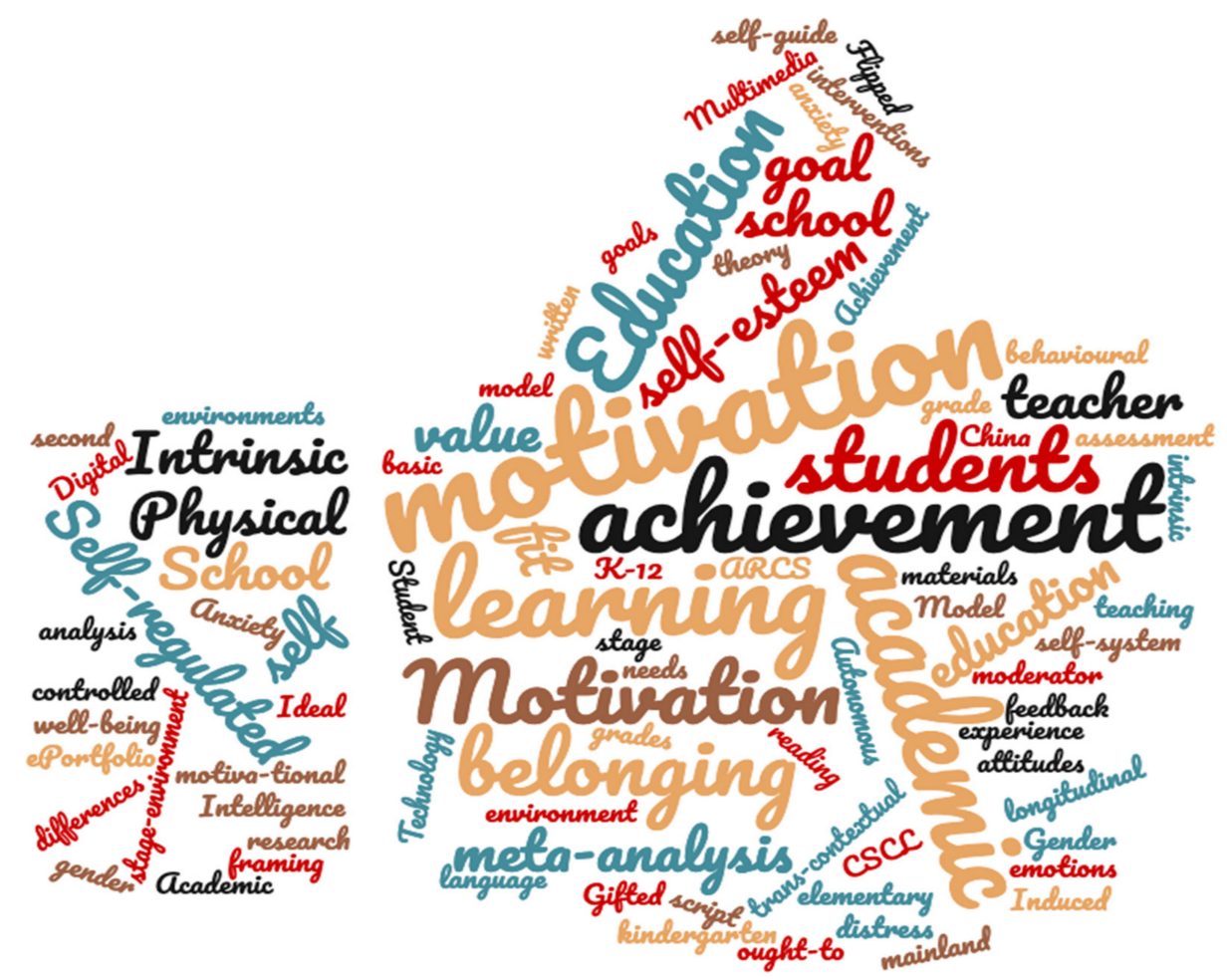

Figure 2. Descriptor cloud of meta-analyses on motivation. 
Table 1. Summary of meta-analysis on motivation in education.

\begin{tabular}{|c|c|c|c|c|c|c|c|}
\hline \multicolumn{8}{|c|}{ Motivation $(n=26)$} \\
\hline Author & Year & Country & Studies (f) & Sample (n) & Purpose & Content & Outcome \\
\hline $\begin{array}{l}\text { Noordzij, Giel and Van } \\
\text { Mierlo [13] }\end{array}$ & 2021 & The Netherlands & 90 & 11,247 & $\begin{array}{l}\text { To study the motivational and } \\
\text { performance effects of } \\
\text { experimentally induced } \\
\text { achievement goals and the } \\
\text { moderating effects of goal } \\
\text { standard and goal framing }\end{array}$ & $\begin{array}{l}\text { Induced achievement } \\
\text { goals, goal standards, } \\
\text { goal framing }\end{array}$ & $\begin{array}{l}\text { Goal framing and goal } \\
\text { standard should be taken } \\
\text { into consideration in } \\
\text { achievement goal research } \\
\text { and practice }\end{array}$ \\
\hline Turhan [15] & 2020 & Turkey & $\begin{array}{c}22 \\
8\end{array}$ & $\begin{array}{l}8010 \\
3017\end{array}$ & $\begin{array}{l}\text { To study the effect of gender } \\
\text { on academic motivation }\end{array}$ & $\begin{array}{l}\text { Gender differences in } \\
\text { academic motivation }\end{array}$ & $\begin{array}{l}\text { Low significant level } \\
\qquad(d=-0.07)\end{array}$ \\
\hline Lei and Chiu [16] & 2020 & China & 96 & - & $\begin{array}{c}\text { To examine Chinese } \\
\text { adolescents' academic } \\
\text { emotions across time via a } \\
\text { cross-temporal meta-analysis }\end{array}$ & $\begin{array}{l}\text { Academic emotions, } \\
\text { mainland } \\
\text { China, students }\end{array}$ & $\begin{array}{c}\text { Gender differences were } \\
\text { not significant }\end{array}$ \\
\hline $\begin{array}{l}\text { Howard, Chong and } \\
\text { Bureau [18] }\end{array}$ & 2020 & Australia & 78 & 41,633 & $\begin{array}{l}\text { To examine covariates } \\
\text { associated with three types of } \\
\text { intrinsic motivation from } \\
\text { self-determination theory } \\
\text { (SDT) within the education } \\
\text { context: intrinsic motivation } \\
\text { (IM) to know, IM to } \\
\text { accomplish, and IM to } \\
\text { experience stimulation }\end{array}$ & $\begin{array}{l}\text { Intrinsic motivation, } \\
\text { education }\end{array}$ & $\begin{array}{l}\text { Intrinsic motivation } \\
\text { appears to be a relatively } \\
\text { homogeneous construct } \\
\text { within educational } \\
\text { psychology }\end{array}$ \\
\hline
\end{tabular}


Table 1. Cont.

\begin{tabular}{|c|c|c|c|c|c|c|c|}
\hline \multicolumn{8}{|c|}{ Motivation $(n=26)$} \\
\hline Author & Year & Country & Studies (f) & Sample (n) & Purpose & Content & Outcome \\
\hline $\begin{array}{l}\text { Slemp, Field and } \\
\text { Cho [19] }\end{array}$ & 2020 & Australia & 98 & 40,253 & $\begin{array}{l}\text { A meta-analysis of teacher } \\
\text { motivation is performed }\end{array}$ & $\begin{array}{l}\text { Autonomous } \\
\text { motivation, } \\
\text { controlled } \\
\text { motivation, teacher, } \\
\text { well-being, distress, } \\
\text { self-determination } \\
\text { theory, basic needs }\end{array}$ & $\begin{array}{l}\text { Autonomous motives } \\
\text { predict teacher well-being } \\
\text { and student supportive } \\
\text { teaching styles }\end{array}$ \\
\hline $\begin{array}{c}\text { Li, Cho, Cosso and } \\
\text { Maeda [20] }\end{array}$ & 2020 & China & 73 & 95,872 & $\begin{array}{l}\text { To examine the association } \\
\text { between K-12 students' } \\
\text { motivation to learn } \\
\text { mathematics and mathematics } \\
\text { anxiety, and explored the } \\
\text { effects of potential } \\
\text { moderating factors }\end{array}$ & $\begin{array}{l}\text { Mathematics anxiety, } \\
\text { motivation for } \\
\text { mathematics, } \\
\text { self-concept, } \\
\text { self-efficacy, intrinsic } \\
\text { value, } \\
\text { achievement value }\end{array}$ & $\begin{array}{l}\text { The strength of the } \\
\text { correlation between } \\
\text { students' mathematics } \\
\text { anxiety with competence } \\
\text { beliefs }(r=-0.48) \text { was } \\
\text { stronger than the } \\
\text { correlation with value } \\
\text { beliefs }(r=-0.36)\end{array}$ \\
\hline $\begin{array}{c}\text { Korpershoek, } \\
\text { Canrinus, } \\
\text { Fokkens-Bruinsma and } \\
\text { Boer [21] }\end{array}$ & 2020 & The Netherlands & 82 & 208,796 & $\begin{array}{l}\text { Examines the relationships } \\
\text { between students' sense of } \\
\text { school belonging and students' } \\
\text { motivational, } \\
\text { social-emotional, behavioural, } \\
\text { and academic functioning in } \\
\text { secondary education }\end{array}$ & $\begin{array}{l}\text { School belonging, } \\
\text { motivation, academic } \\
\text { achievement, school } \\
\text { belonging, } \\
\text { meta-analysis }\end{array}$ & $\begin{array}{l}\text { The results reveal that } \\
\text { school belonging plays an } \\
\text { important role in students' } \\
\text { school life }\end{array}$ \\
\hline $\begin{array}{l}\text { Radkowitsh, Vogel and } \\
\text { Fisher [22] }\end{array}$ & 2020 & Germany & 53 & 5616 & $\begin{array}{c}\text { To study the effect of learning } \\
\text { with a computer-supported } \\
\text { collaboration (CSCL) script on } \\
\text { unguided } \\
\text { collaborative learning }\end{array}$ & $\begin{array}{l}\text { CSCL script, learning, } \\
\text { collaborative, } \\
\text { motivation }\end{array}$ & $\begin{array}{l}\text { CSCL scripts leads to a } \\
\text { non-significant positive } \\
\text { effect on motivation } \\
\left(\text { Hedges' }^{\prime} g=0.13\right)\end{array}$ \\
\hline $\begin{array}{l}\text { Zheng, Bhagat, Zhen } \\
\text { and Zhang [23] }\end{array}$ & 2020 & Taiwan & 95 & 15,386 & $\begin{array}{l}\text { To examine the overall } \\
\text { effectiveness of the flipped } \\
\text { classroom on students' } \\
\text { learning achievement } \\
\text { and motivation }\end{array}$ & $\begin{array}{l}\text { Flipped classroom, } \\
\text { motivation, learning }\end{array}$ & $\begin{array}{l}\text { Flipped classroom } \\
\text { approach had a moderate } \\
\text { effect size for } \\
\text { learning motivation }\end{array}$ \\
\hline
\end{tabular}


Table 1. Cont.

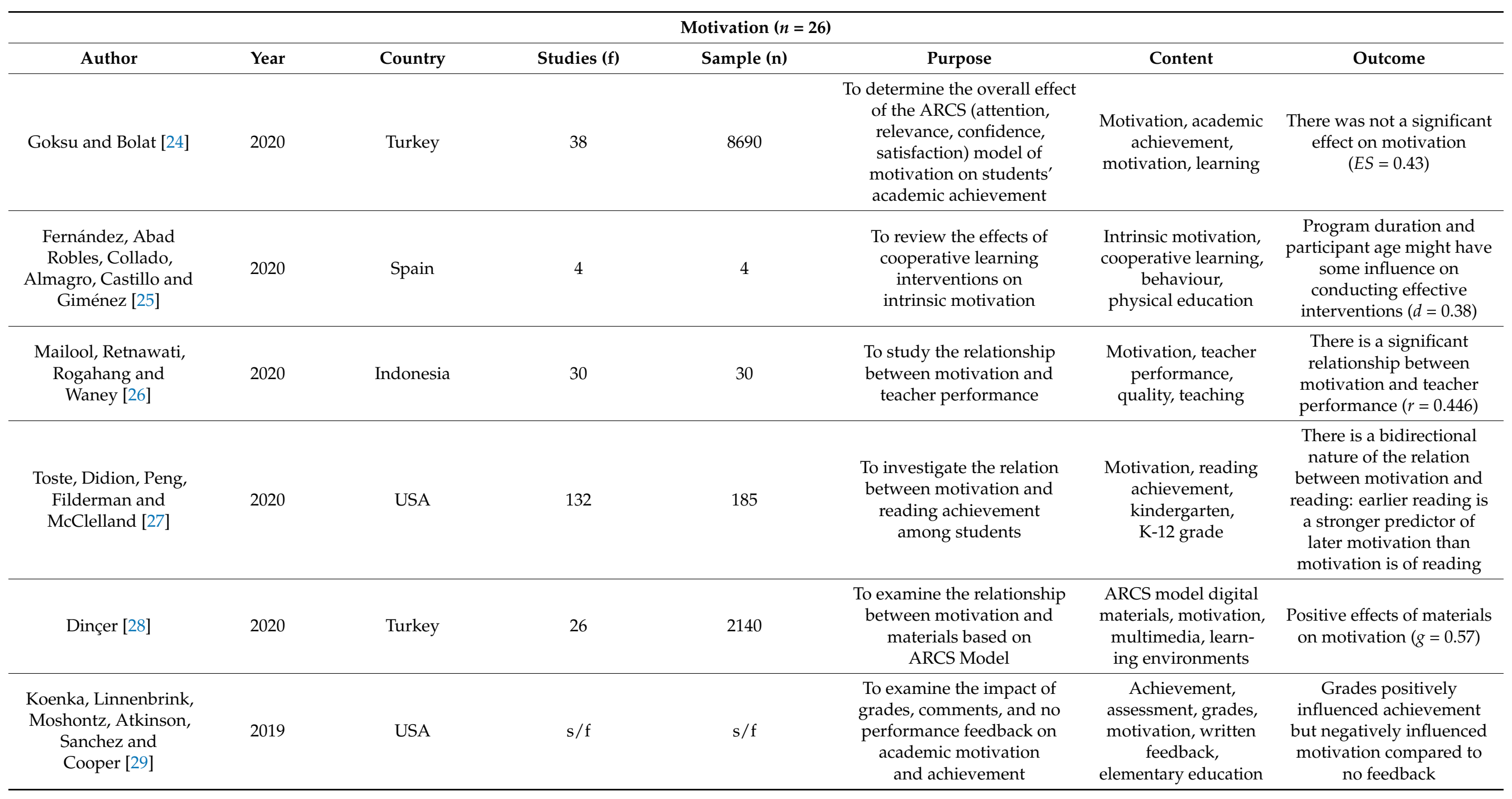


Table 1. Cont.

\begin{tabular}{|c|c|c|c|c|c|c|c|}
\hline \multicolumn{8}{|c|}{ Motivation $(n=26)$} \\
\hline Author & Year & Country & Studies (f) & Sample (n) & Purpose & Content & Outcome \\
\hline $\begin{array}{l}\text { Kriegbaum, Becker and } \\
\text { Spinath [30] }\end{array}$ & 2018 & Germany & 74 & 80,145 & $\begin{array}{l}\text { To study the predictive power } \\
\text { of intelligence and motivation } \\
\text { for school achievement }\end{array}$ & $\begin{array}{l}\text { Intelligence, } \\
\text { motivation, school } \\
\text { achievement }\end{array}$ & $\begin{array}{l}\text { Both intelligence and } \\
\text { motivation contribute to } \\
\text { the prediction of school } \\
\text { achievement }\end{array}$ \\
\hline Volk [31] & 2018 & Saudi Arabia & 32 & 32,078 & $\begin{array}{l}\text { Meta-analysis of the L2 } \\
\text { motivational self-system }\end{array}$ & $\begin{array}{l}\text { Ideal L2 self, } \\
\text { ought-to L2 self, L2 } \\
\text { learning experience, } \\
\text { L2 motivational } \\
\text { self-system, } \\
\text { self-guide }\end{array}$ & $\begin{array}{l}\text { The ideal L2 self, the } \\
\text { ought-to L2 self, and the } \\
\text { L2 learning experience } \\
\text { were significant predictors } \\
\text { of subjective } \\
\text { intended effort }\end{array}$ \\
\hline Murthy and Jiar [32] & 2017 & Malaysia & 19 & - & $\begin{array}{l}\text { To examine the effects among } \\
\text { students' anxiety, motivation } \\
\text { and attitudes in learning } \\
\text { English as a second language }\end{array}$ & $\begin{array}{l}\text { Anxiety, attitudes, } \\
\text { learning, second } \\
\text { language, motivation }\end{array}$ & $\begin{array}{l}\text { There are strong } \\
\text { relationships among these } \\
\text { three variables }\end{array}$ \\
\hline $\begin{array}{c}\text { Higgins, } \\
\text { Huscroft-Dangelo and } \\
\text { Crawford [33] }\end{array}$ & 2017 & USA & 24 & 4522 & $\begin{array}{c}\text { To determine the effects of } \\
\text { technology use on student } \\
\text { achievement, motivation, and } \\
\text { attitude }\end{array}$ & $\begin{array}{l}\text { Technology, } \\
\text { achievement, } \\
\text { motivation }\end{array}$ & $\begin{array}{c}\text { Significant overall impact } \\
\text { of technology on student } \\
\text { achievement, motivation, } \\
\text { and attitudes }\end{array}$ \\
\hline Ergen and Kanadli [34] & 2017 & Turkey & 21 & 5353 & $\begin{array}{c}\text { To study the effect of } \\
\text { self-regulated earning } \\
\text { strategies on academic } \\
\text { achievement }\end{array}$ & $\begin{array}{l}\text { Self-regulated } \\
\text { learning, academic } \\
\text { achievement, } \\
\text { moderator analysis }\end{array}$ & $\begin{array}{l}\text { Self-regulated learning } \\
\text { strategies had a "large" } \\
\text { effect }(d=0.859) \text { on } \\
\text { academic achievement }\end{array}$ \\
\hline $\begin{array}{l}\text { Lazowski and } \\
\text { Hulleman [35] }\end{array}$ & 2016 & USA & 34 & 38,377 & $\begin{array}{c}\text { To study the interventions in } \\
\text { education in relation to } \\
\text { motivation theory }\end{array}$ & $\begin{array}{l}\text { Education } \\
\text { interventions, } \\
\text { motivation }\end{array}$ & $\begin{array}{l}\text { Interventions were } \\
\text { generally effective } \\
\qquad(d=0.49)\end{array}$ \\
\hline $\begin{array}{c}\text { Hagger and } \\
\text { Chatzisarantis [36] }\end{array}$ & 2016 & USA & 10 & 10 & $\begin{array}{l}\text { To study a proposal for a } \\
\text { trans-contextual model in } \\
\text { physical education }\end{array}$ & $\begin{array}{l}\text { Motivation, } \\
\text { trans-contextual } \\
\text { model, physical } \\
\text { education }\end{array}$ & $\begin{array}{l}\text { There are important gaps } \\
\text { in the results. The } \\
\text { significant relationship is } \\
\text { not sufficiently clear }\end{array}$ \\
\hline
\end{tabular}


Table 1. Cont.

\begin{tabular}{|c|c|c|c|c|c|c|c|}
\hline \multicolumn{8}{|c|}{ Motivation $(n=26)$} \\
\hline Author & Year & Country & Studies (f) & Sample (n) & Purpose & Content & Outcome \\
\hline Bak [37] & 2016 & Korea & 80 & 684 & $\begin{array}{l}\text { To examine the differences } \\
\text { between gifted and non-gifted } \\
\text { students on } \\
\text { academic motivation }\end{array}$ & $\begin{array}{l}\text { Gifted, academic } \\
\text { motivation, students }\end{array}$ & $\begin{array}{l}\text { The overall mean effect } \\
\text { size of positive academic } \\
\text { motivation was } 0.705 \\
\text { above moderate effect }\end{array}$ \\
\hline Maeng [38] & 2016 & Korean & 74 & 175 & $\begin{array}{l}\text { To study the effect of } \\
\text { motivation on L2 learning } \\
\text { in Korea }\end{array}$ & $\begin{array}{l}\text { L2 learning, } \\
\text { motivation, students }\end{array}$ & $\begin{array}{l}\text { The results show a weak } \\
\text { correlation between L2 } \\
\text { proficiency and motivation, } \\
\text { L2 strategy and motivation, } \\
\text { and psychological } \\
\text { concepts and motivation. }\end{array}$ \\
\hline
\end{tabular}

Table 2. Summary of meta-analysis on evaluation/assessment in education.

\begin{tabular}{|c|c|c|c|c|c|c|c|}
\hline \multicolumn{8}{|c|}{ Evaluation/Assessment $(n=28)$} \\
\hline Author & Year & Country & Studies (f) & Sample (n) & Purpose & Content & Outcome \\
\hline $\begin{array}{l}\text { Zheng, Bhagat, Zhen } \\
\text { and Zhang [23] }\end{array}$ & 2020 & China & 37 & - & $\begin{array}{l}\text { To study the effects of } \\
\text { technology-facilitated peer } \\
\text { assessment based on two main } \\
\text { elements: (1) technology- } \\
\text { facilitated peer assessment, } \\
\text { (2) the use of extra supporting } \\
\text { strategies in } \\
\text { technology-facilitated } \\
\text { peer assessment }\end{array}$ & $\begin{array}{c}\text { Peer assessment; } \\
\text { learning achievement }\end{array}$ & $\begin{array}{c}\text { Results indicated that } \\
\text { technology-facilitated peer } \\
\text { assessment had a } \\
\text { significant and medium } \\
\text { effect on learning } \\
\text { achievements with an } \\
\text { overall mean effect size of } \\
\qquad d=0.576\end{array}$ \\
\hline $\begin{array}{l}\text { Rios, Ihlenfeldt, } \\
\text { Chavez [39] }\end{array}$ & 2020 & USA & 26 & 11,069 & $\begin{array}{l}\text { To study the effectiveness of } \\
\text { accommodations for English } \\
\text { language learners on } \\
\text { evidence-based state } \\
\text { accountability assessments }\end{array}$ & $\begin{array}{l}\text { English learner, } \\
\text { accountability } \\
\text { assessments, } \\
\text { performance, } \\
\text { effectiveness }\end{array}$ & $\begin{array}{l}\text { Results suggest that } \\
\text { currently employed EL test } \\
\text { accommodations lack } \\
\text { evidence of their } \\
\text { effectiveness }\end{array}$ \\
\hline
\end{tabular}


Table 2. Cont.

\begin{tabular}{|c|c|c|c|c|c|c|c|}
\hline \multicolumn{8}{|c|}{ Evaluation/Assessment $(\mathrm{n}=\mathbf{2 8})$} \\
\hline Author & Year & Country & Studies (f) & Sample (n) & Purpose & Content & Outcome \\
\hline $\begin{array}{l}\text { Li, Xiong, Hunter, Guo } \\
\text { and Tywoniw [40] }\end{array}$ & 2020 & USA & 58 & - & $\begin{array}{l}\text { To study whether peer } \\
\text { assessment promotes } \\
\text { student's learning }\end{array}$ & $\begin{array}{l}\text { Peer assessment, } \\
\text { learning, students }\end{array}$ & $\begin{array}{l}\text { The most critical factor is } \\
\text { rater training. When } \\
\text { students receive rater } \\
\text { training, the effect size of } \\
\text { peer assessment is } \\
\text { substantially larger than } \\
\text { when students do not } \\
\text { receive such training }\end{array}$ \\
\hline $\begin{array}{l}\text { Lee, Chung, Zhang, } \\
\text { Abedi and } \\
\text { Warschauer [41] }\end{array}$ & 2020 & USA & 33 & 33 & $\begin{array}{l}\text { To review empirical studies } \\
\text { that conducted formative } \\
\text { assessment interventions to } \\
\text { improve student learning }\end{array}$ & $\begin{array}{c}\text { Formative } \\
\text { assessment, learning }\end{array}$ & $\begin{array}{l}\text { Small-sized positive effect } \\
\text { of formative assessment on } \\
\text { student learning }(d=0.29)\end{array}$ \\
\hline $\begin{array}{l}\text { Double, MoGrane and } \\
\text { Hopfenbeck [42] }\end{array}$ & 2020 & England & 54 & - & $\begin{array}{l}\text { To examine the impact of peer } \\
\text { assessment on } \\
\text { academic performance }\end{array}$ & $\begin{array}{l}\text { Effectiveness, peer } \\
\text { assessment, } \\
\text { academic performance }\end{array}$ & $\begin{array}{l}\text { Results suggested that the } \\
\text { effectiveness of peer } \\
\text { assessment was } \\
\text { remarkably robust across a } \\
\text { wide range of contexts }\end{array}$ \\
\hline $\begin{array}{l}\text { Mortaz, Jalili, } \\
\text { Masoomi, Shirazi, } \\
\text { Nedjat and } \\
\text { Norcini [43] }\end{array}$ & 2020 & Iran and Sweden & 58 & - & $\begin{array}{l}\text { To examine the utility of the } \\
\text { mini-clinical evaluation } \\
\text { exercise (CEX) for assessing } \\
\text { undergraduate and } \\
\text { postgraduate medical trainees }\end{array}$ & $\begin{array}{l}\text { Evaluation, education } \\
\text { quality, clinical } \\
\text { competence, education } \\
\text { medical, education } \\
\text { physical examination }\end{array}$ & $\begin{array}{l}\text { The mini-CEX improved } \\
\text { students' performance in } \\
\text { other examinations }\end{array}$ \\
\hline $\begin{array}{l}\text { Hanshaw and } \\
\text { Dickerson [44] }\end{array}$ & 2020 & USA & 20 & - & $\begin{array}{c}\text { What is the effect of science on } \\
\text { the evidence of learning } \\
\text { outcomes in high-fidelity } \\
\text { simulation in undergraduate } \\
\text { nursing education? }\end{array}$ & $\begin{array}{l}\text { Learning outcomes, } \\
\text { evaluation, evidence, } \\
\text { skills, simulation }\end{array}$ & $\begin{array}{l}\text { Outcome measures } \\
\text { reviewed include } \\
\text { increasing levels } \\
\text { of thinking }\end{array}$ \\
\hline Lai and Bower [45] & 2020 & Australia & 73 & - & $\begin{array}{l}\text { To analyse the impact of } \\
\text { learning technology use across } \\
\text { different aspects of evaluation }\end{array}$ & $\begin{array}{l}\text { Evaluation, learning } \\
\text { technology, tertiary }\end{array}$ & $\begin{array}{c}\text { Interaction, gamification, } \\
\text { constructivism, } \\
\text { student-centred learning } \\
\text { and feedback are effective } \\
\text { approaches to learning }\end{array}$ \\
\hline
\end{tabular}


Table 2. Cont.

\begin{tabular}{|c|c|c|c|c|c|c|c|}
\hline \multicolumn{8}{|c|}{ Evaluation/Assessment $(n=28)$} \\
\hline Author & Year & Country & Studies (f) & Sample (n) & Purpose & Content & Outcome \\
\hline $\begin{array}{c}\text { Zhao, Xu, Jiang and } \\
\text { Ding [46] }\end{array}$ & 2020 & China & 15 & 816 & $\begin{array}{l}\text { To study the performance of } \\
\text { virtual reality (VR) } \\
\text { anatomy education }\end{array}$ & $\begin{array}{l}\text { Augmented and virtual } \\
\text { reality, improving } \\
\text { classroom, teaching, } \\
\text { learning strategies }\end{array}$ & $\begin{array}{l}\text { The finding confirms that } \\
\text { VR may act as an efficient } \\
\text { way to improve the } \\
\text { learners' level of } \\
\text { anatomy knowledge }\end{array}$ \\
\hline $\begin{array}{c}\text { Lester, Chow and } \\
\text { Melton [47] }\end{array}$ & 2020 & USA & 30 & - & $\begin{array}{l}\text { To extend the literature by } \\
\text { meta-analysing a } \\
\text { comprehensive sample of } \\
\text { studies and examine the } \\
\text { impact of study quality on } \\
\text { meta-analytical results }\end{array}$ & $\begin{array}{l}\text { Academic success, } \\
\text { adolescent education, } \\
\text { program evaluation }\end{array}$ & $\begin{array}{l}\text { The inclusion of lower } \\
\text { quality studies } \\
\text { significantly impacts } \\
\text { overall outcomes in } \\
\text { comparison to prior } \\
\text { meta-analyses that have } \\
\text { limited samples }\end{array}$ \\
\hline $\begin{array}{l}\text { Reyes, Dinh, Lacerenza } \\
\text { and Salas [48] }\end{array}$ & 2020 & USA & 73 & 5654 & $\begin{array}{l}\text { Effectiveness of educational } \\
\text { leadership development (LD) } \\
\text { in students }\end{array}$ & $\begin{array}{l}\text { Leadership, education, } \\
\text { effectiveness, } \\
\text { higher education }\end{array}$ & $\begin{array}{l}\text { LD programs within } \\
\text { higher education work }\end{array}$ \\
\hline $\begin{array}{c}\text { Husiman, Saab, Van } \\
\text { den Broek and Van } \\
\text { Driel [50] }\end{array}$ & 2019 & The Netherlands & 24 & - & $\begin{array}{l}\text { To analyse the impact that } \\
\text { peer feedback has on students' } \\
\text { writing performance }\end{array}$ & $\begin{array}{l}\text { Peer feedback, peer } \\
\text { assessment, } \\
\text { academic writing }\end{array}$ & $\begin{array}{l}\text { Formative peer feedback } \\
\text { has significant values }\end{array}$ \\
\hline $\begin{array}{l}\text { Gegenfurtner and } \\
\text { Ebner [51] }\end{array}$ & 2019 & Germany & 5 & 381 & $\begin{array}{l}\text { To study the effect sizes and } \\
\text { test the predictive validity of } \\
\text { Kirkpatrick's assumption }\end{array}$ & $\begin{array}{c}\text { Adult learning, } \\
\text { computer-mediated, } \\
\text { communication, distance } \\
\text { education and } \\
\text { telelearning, distributed } \\
\text { learning environments, } \\
\text { media in education }\end{array}$ & $\begin{array}{c}\text { Kirkpatrick's model does } \\
\text { not produce the expected } \\
\text { effects on learning }\end{array}$ \\
\hline
\end{tabular}


Table 2. Cont.

\begin{tabular}{|c|c|c|c|c|c|c|c|}
\hline \multicolumn{8}{|c|}{ Evaluation/Assessment $(n=28)$} \\
\hline Author & Year & Country & Studies (f) & Sample (n) & Purpose & Content & Outcome \\
\hline Hurwitz [52] & 2019 & USA & 45 & 24,624 & $\begin{array}{l}\text { To examine the effects of ready } \\
\text { to learn (RTL) }\end{array}$ & $\begin{array}{l}\text { Child, learning, literacy, } \\
\text { preschool child program, } \\
\text { evaluation, } \\
\text { reading, television }\end{array}$ & $\begin{array}{l}\text { Results indicate positive } \\
\text { effects of RTL media } \\
\text { exposure on children's } \\
\text { literacy outcomes, } \\
\text { especially vocabulary and } \\
\text { phonological concepts }\end{array}$ \\
\hline $\begin{array}{l}\text { Castro and } \\
\text { Tumibay [53] }\end{array}$ & 2019 & Philippines & 50 & - & $\begin{array}{l}\text { To examine the efficacy of } \\
\text { online learning in } \\
\text { educating students }\end{array}$ & $\begin{array}{l}\text { ADDIE (analysis, design, } \\
\text { development, } \\
\text { implementation, and } \\
\text { evaluation) framework, } \\
\text { efficacy, online } \\
\text { learning course }\end{array}$ & $\begin{array}{l}\text { The efficacy of online } \\
\text { learning courses through } \\
\text { having well-planned, } \\
\text { well-designed courses and } \\
\text { programs for higher } \\
\text { education institution }\end{array}$ \\
\hline $\begin{array}{c}\text { Koenka, } \\
\text { Linnenbrink-Garcia, } \\
\text { Moshontz, Atkinson, } \\
\text { Sanchez and } \\
\text { Cooper [29] }\end{array}$ & 2019 & USA & - & - & $\begin{array}{l}\text { To examine the impact of } \\
\text { grades, comments, and no } \\
\text { performance feedback on } \\
\text { academic motivation and } \\
\text { achievement in elementary } \\
\text { and secondary school }\end{array}$ & $\begin{array}{l}\text { Motivation, performance, } \\
\text { achievement, } \\
\text { secondary school }\end{array}$ & $\begin{array}{l}\text { The overall effects varied } \\
\text { as a function of the type of } \\
\text { motivation }\end{array}$ \\
\hline Petersen [54] & 2018 & USA & 16 & - & $\begin{array}{l}\text { To examine the gender } \\
\text { differences in US state } \\
\text { verbal assessments }\end{array}$ & $\begin{array}{c}\text { Gender, verbal } \\
\text { performance, } \\
\text { performance assessments }\end{array}$ & $\begin{array}{l}\text { The small gender } \\
\text { differences in verbal } \\
\text { performance increased in a } \\
\text { linear pattern from grades } \\
3 \text { to } 8 \text { and then remained } \\
\text { steady in high school } \\
(d=0.29)\end{array}$ \\
\hline $\begin{array}{l}\text { Maan, Hussain and } \\
\text { Sharma [55] }\end{array}$ & 2018 & India & - & - & $\begin{array}{c}\text { To find the existing process of } \\
\text { curricular delivery of physical } \\
\text { education program }\end{array}$ & $\begin{array}{l}\text { Sport, benchmarking, } \\
\text { university, } \\
\text { teaching, evaluation }\end{array}$ & $\begin{array}{l}\text { The physical education } \\
\text { curriculum differs from } \\
\text { country to country and } \\
\text { within the institutions in } \\
\text { the country (India) }\end{array}$ \\
\hline
\end{tabular}


Table 2. Cont.

\begin{tabular}{|c|c|c|c|c|c|c|c|}
\hline \multicolumn{8}{|c|}{ Evaluation/Assessment $(n=28)$} \\
\hline Author & Year & Country & Studies (f) & Sample (n) & Purpose & Content & Outcome \\
\hline $\begin{array}{l}\text { Hamad, Elser, Tran, } \\
\text { Rehkopf and } \\
\text { Goodman [56] }\end{array}$ & 2018 & USA & 25 & 600 & $\begin{array}{l}\text { The association between } \\
\text { increased educational } \\
\text { attainment and } \\
\text { improved health }\end{array}$ & $\begin{array}{l}\text { Compulsory schooling, } \\
\text { educational attainment } \\
\text { instrumental, } \\
\text { policy evaluation }\end{array}$ & $\begin{array}{l}\text { The meta-analysis } \\
\text { demonstrated small } \\
\text { beneficial effects for } \\
\text { mortality, smoking, } \\
\text { and obesity }\end{array}$ \\
\hline $\begin{array}{l}\text { Cui, Li, Geng, Zhang } \\
\text { and Jin [57] }\end{array}$ & 2018 & China & 9 & 1079 & $\begin{array}{l}\text { To assess the effectiveness of } \\
\text { evidence-based nursing (EBN) } \\
\text { on the development of critical } \\
\text { thinking for nursing students }\end{array}$ & $\begin{array}{l}\text { Critical thinking } \\
\text { evidence-based nursing } \\
(\mathrm{EBN}), \text { nursing education }\end{array}$ & $\begin{array}{l}\text { The effectiveness of } \\
\text { evidence-based nursing } \\
\text { was superior to that of } \\
\text { traditional teaching on } \\
\text { nursing students' } \\
\text { critical thinking }\end{array}$ \\
\hline $\begin{array}{c}\text { Panadero, Jonsson and } \\
\text { Botella [58] }\end{array}$ & 2017 & Spain & 19 & 2305 & $\begin{array}{l}\text { To study the effects of } \\
\text { self-assessment on students' } \\
\text { self-regulated learning (SRL) } \\
\quad \text { and self-efficacy }\end{array}$ & $\begin{array}{l}\text { Self-assessment, learning, } \\
\text { self-efficacy }\end{array}$ & $\begin{array}{l}\text { The importance of } \\
\text { self-assessment } \\
\text { interventions to promote } \\
\text { students' use of learning } \\
\text { strategies and its effects on } \\
\text { motivational variables, } \\
\text { such as self-efficacy }\end{array}$ \\
\hline Conn [59] & 2017 & USA & 56 & 345 & $\begin{array}{l}\text { To identify educational } \\
\text { interventions with an impact } \\
\text { on student learning in } \\
\text { Sub-Saharan Africa }\end{array}$ & $\begin{array}{l}\text { Education and } \\
\text { developing countries, } \\
\text { impact evaluation }\end{array}$ & $\begin{array}{l}\text { Pedagogical programs that } \\
\text { employed adaptive } \\
\text { instruction or teacher } \\
\text { coaching were particularly } \\
\text { effective }\end{array}$ \\
\hline Vo, Zhu and Diep [60] & 2017 & $\begin{array}{l}\text { Belgium and } \\
\text { VietNam }\end{array}$ & 34 & 35,690 & $\begin{array}{l}\text { To analyse the impact of } \\
\text { blended learning (BL) on the } \\
\text { academic achievement of } \\
\text { higher education students }\end{array}$ & $\begin{array}{c}\text { Blended learning } \\
\text { discipline, higher } \\
\text { education, student } \\
\text { performance }\end{array}$ & $\begin{array}{c}\text { The effect of BL in STEM } \\
\text { disciplines is significantly } \\
\text { higher than that of } \\
\text { non-STEM disciplines }\end{array}$ \\
\hline
\end{tabular}


Table 2. Cont.

\begin{tabular}{|c|c|c|c|c|c|c|c|}
\hline \multicolumn{8}{|c|}{ Evaluation/Assessment $(n=28)$} \\
\hline Author & Year & Country & Studies (f) & Sample (n) & Purpose & Content & Outcome \\
\hline $\begin{array}{c}\text { Yue, Zhang, Zhang and } \\
\text { Jin [61] }\end{array}$ & 2017 & China & Not access & Not access & $\begin{array}{l}\text { The review and meta-analysis } \\
\text { were to assess the effect of } \\
\text { concept mapping on } \\
\text { developing critical thinking in } \\
\text { nursing education. }\end{array}$ & $\begin{array}{l}\text { Concept mapping, } \\
\text { critical thinking, } \\
\text { education }\end{array}$ & $\begin{array}{l}\text { The concept mapping } \\
\text { could affect the critical } \\
\text { thinking affective } \\
\text { dispositions and critical } \\
\text { thinking cognitive skills }\end{array}$ \\
\hline $\begin{array}{c}\text { Li, Xiong, Zang, } \\
\text { Kornhaber, Lyu, } \\
\text { Chung and Suen [62] }\end{array}$ & 2016 & USA & 69 & 69 & $\begin{array}{l}\text { To study the effect of } \\
\text { computer-assisted peer } \\
\text { assessment on student } \\
\text { performance }\end{array}$ & $\begin{array}{l}\text { Assessment, } \\
\text { computer-assisted, } \\
\text { performance }\end{array}$ & It is moderately strong \\
\hline Sung, Chang and Liu & 2016 & Taiwan & 110 & 4121 & $\begin{array}{l}\text { To study the effects of } \\
\text { integrated mobile devices on } \\
\text { teaching and learning }\end{array}$ & $\begin{array}{c}\text { Evaluation } \\
\text { methodologies, } \\
\text { pedagogical issues, } \\
\text { teaching, learning } \\
\text { strategies }\end{array}$ & $\begin{array}{l}\text { A moderate mean effect } \\
\text { size of } d=0.523\end{array}$ \\
\hline $\begin{array}{c}\text { Zhou, Zhou, Huang, } \\
\text { Xu, Zhang, Zeng and } \\
\text { Qian [64] }\end{array}$ & 2016 & China & 16 & 1826 & $\begin{array}{c}\text { A critical overview of } \\
\text { problem-based learning (PBL) } \\
\text { practices in Chinese } \\
\text { pharmacy education }\end{array}$ & $\begin{array}{c}\text { Pedagogics, } \\
\text { problem-based learning, } \\
\text { questionnaire, skill, } \\
\text { teaching, theoretical } \\
\text { model, university } \\
\text { education, } \\
\text { problem-based } \\
\text { learning, evaluation }\end{array}$ & $\begin{array}{c}\text { PBL pedagogy is superior } \\
\text { to traditional lecture-based } \\
\text { teaching in Chinese } \\
\text { pharmacy education }\end{array}$ \\
\hline
\end{tabular}




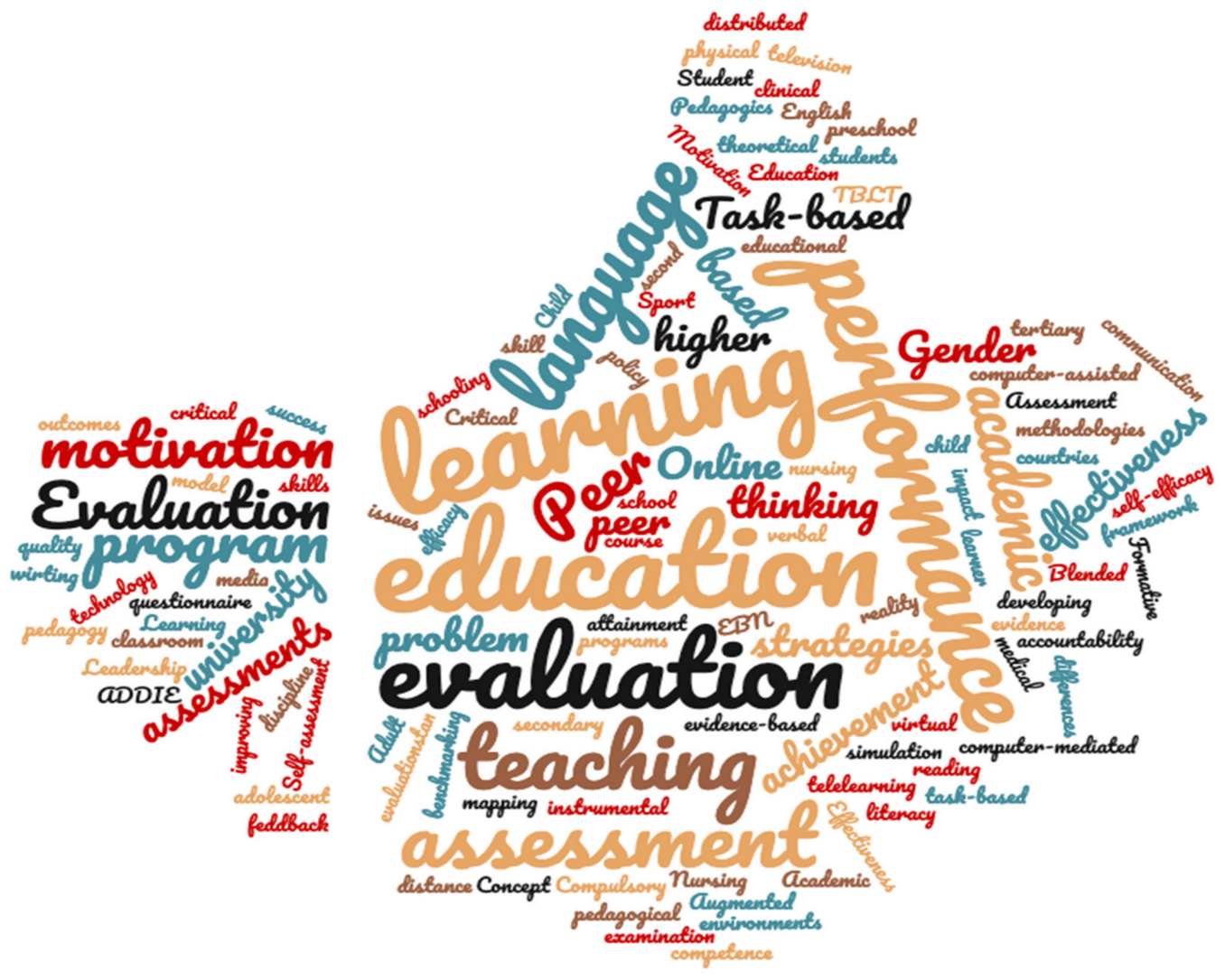

Figure 3. Descriptor cloud of meta-analyses on evaluation or assessment.

\section{Discussion and Conclusions}

This article highlighted the importance of motivation and evaluation to our understanding of educational processes from a sustainable perspective. In other words, there is evidence that both topics are of interest to the scientific community, either because of the number of publications or the diversity of aspects they deal with. This was our intention from the outset. However, in addition, this map allows us to analyse motivation and evaluation in the current context in relation to the priorities, absences and possibilities for the development of research in education.

These issues point to at least two possible directions for further research: (1) the need for more meta-analyses covering the range of topics on motivation and evaluation; (2) the absence of publications undertaking a stated and defined perspective of the sustainability of educational processes is highlighted. There are no meta-analyses that study motivation from evaluation and evaluation from motivation guided by the perspective of the sustainability of educational processes. This is undoubtedly a manifest absence.

In sum, motivation and evaluation are key to promoting successful educational processes for all. These processes must be sustainable and must contribute to the sustainability of education. Despite the remarkable increase in meta-analyses on these topics, it is evident that there is a lack of a sustainable perspective, most likely the result of experimental studies that provide evidence in this area.

The review that we carried out must also be understood in terms of its limitations: (1) It focused on meta-analyses as a criterion of usefulness and priority in the generation of knowledge based on scientific evidence, but there are a large number of other valuable works. (2) Three of all existing databases were used. It is true that these three databases are of high quality, and the impact of international publications is included in them. (3) The use of PICO (adapted according to the nature of the study) is a guarantee of the quality of the work procedure, as well as PRISMA and the other tools used. 
Author Contributions: Conceptualization, J.S.-S. and D.H.-A.; methodology, J.S.-S.; B.I.B.-C. and F.-M.L.-G.; software, J.S.-S.; validation, B.I.B.-C. and F.-M.L.-G.; formal analysis, J.S.-S.; F.-M.L.-G. and B.I.B.-C.; investigation, J.S.-S.; B.I.B.-C. and D.H.-A.; resources, F.-M.L.-G.; data curation, J.S.-S.; writing-original draft preparation, J.S.-S. and F.-M.L.-G.; writing-review and editing, J.S.-S. and D.H.-A.; visualization, D.H.-A. and B.I.B.-C.; supervision, J.S.-S. All authors have read and agreed to the published version of the manuscript.

Funding: This research received no external funding.

Institutional Review Board Statement: Not applicable for studies not involving humans or animals.

Informed Consent Statement: Not applicable for studies not involving humans or animals.

Data Availability Statement: The nature of the study (review) already implies in itself unique and complementary material. There is no need to attach further material.

Acknowledgments: We are grateful to the University of Castilla-La Mancha for the use of the officially registered databases.

Conflicts of Interest: The authors declare no conflict of interest.

\section{References}

1. United Nations. Transforming Our World: The 2030 Agenda for Sustainable Development; UN: New York, NY, USA, 2015.

2. Calvente, A.M. El Concepto Moderno de Sustentabilidad. UAIS Sustentabilidad 2007, 1, 1-7.

3. Grosseck, G.; Tîru, L.G.; Bran, R.A. Education for sustainable development: Evolution and perspectives: A bibliometric review of research, 1992-2018. Sustainability 2019, 11, 6136. [CrossRef]

4. Hargreaves, A.; Fink, D. Sustainable Leadership; Jossey-Bass: San Francisco, CA, USA, 2006.

5. Sekhar, C.; Patwardhan, M.; Kumar, R. A literature review on motivation. Glob. Bus. Perspect. 2013, 1, 471-487. [CrossRef]

6. Hortigüela-Alcalá, D.; Sánchez-Santamaría, J.; Pérez-Pueyo, Á.; Abella-García, V. Social networks to promote motivation and learning in higher education from the students' perspective. Inno. Educ. Teach. Inter. 2019, 56, 412-422. [CrossRef]

7. Murchan, D.; Shiel, G. Understanding and Applying Assessment in Education; Sage: London, UK, 2017.

8. López-Pastor, V.M.; Pérez-Pueyo, Á. Buenas Prácticas Docentes: Evaluación Formativa y Compartida en Educación: Experiencias de Éxito en Todas las Etapas Educativas; Universidad de León: León, Spain, 2017.

9. Zawacki-Richter, O.; Kerres, M.; Bedenlier, S.; Bond, M.; Buntins, K. Systematic Reviews in Educational Research: Methodology, Perspectives and Application, 1st ed.; Spring Nature: Wiesbaden, Germany, 2020; pp. 1-161.

10. Moher, D.; Liberati, A.; Tetzlaff, J.; Altman, D.G. The PRISMA Group. Preferred Reporting Items for Systematic Reviews and Meta-Analyses: The PRISMA Statement. PLoS Med. 2009, 6, 336-341. [CrossRef]

11. Reeves, B.; Wells, G.; Thuku, M.; Hamel, C.; Moran, J.; Henry, D. AMSTAR 2: A critical appraisal tool for systematic reviews that include randomized or non-randomised studies of healthcare interventions, or both. BMJ 2017, 358, 1-9. [CrossRef]

12. Moher, D.; Schulz, K.F.; Altman, D. The CONSORT statement: Revised recommendations for improving the quality of reports of parallel-group randomized trials. JAMA 2001, 285, 1987-1991. [CrossRef] [PubMed]

13. Noordzij, G.; Lisenne, G.; Van Mierlo, H. A meta-analysis of induced achievement goals: The moderating effects of goal standard and goal framing. Soc. Psychol. Educ. 2021, 24, 195-245. [CrossRef]

14. Segaran, M.; Zuwati, H. Self-regulated learning through ePortfolio: A meta-analysis. Malays. J. Learn. Instr. 2021, 18, 131-156. [CrossRef]

15. Turhan, N.S. Gender differences in academic motivation: A meta-analysis. IJPES 2020, 7, 211-224. [CrossRef]

16. Lei, H.; Yunhuo, C.; Ming, C. The relationship between teacher support and students' academic emotions: A meta-analysis. Front. Psychol. 2018, 8, 2288. [CrossRef]

17. Scherrer, C.; Preckel, F. Development of motivational variables and self-esteem during the school career: A meta-analysis of longitudinal studies. Rev. Educ. Res. 2019, 89, 211-258. [CrossRef]

18. Howard, J.L.; Jane, X.Y.; Bureau, J. The tripartite model of intrinsic motivation in education: A 30-year retrospective and meta-analysis. J. Personal. 2020, 88, 1268-1285. [CrossRef] [PubMed]

19. Slemp, G.R.; Field, J.; Cho, A. A meta-analysis of autonomous and controlled forms of teacher motivation. J. Vocat. Behav. 2020, 121, 103459. [CrossRef]

20. Li, Q.; Cho, H.; Cosso, J.; Maeda, Y. Relations Between Students' Mathematics Anxiety and Motivation to Learn Mathematics: A Meta-Analysis. Educ. Psychol. Rev. 2021, 1-33. [CrossRef]

21. Korpershoek, H.; Canrinus, E.T.; Fokkens-Bruinsma, M.; de Boer, H. The relationships between school belonging and students' motivational, social-emotional, behavioural, and academic outcomes in secondary education: A meta-analytic review. Res. Pap. Educ. 2020, 35, 641-680. [CrossRef]

22. Radkowitsch, A.; Vogel, F.; Fischer, F. Good for learning, bad for motivation? A meta-analysis on the effects of computer-supported collaboration scripts. IJCSCL 2020, 15, 5-47. [CrossRef] 
23. Zheng, L.; Bhagat, K.K.; Zhen, Y.; Zhang, X. The effectiveness of the flipped classroom on students' learning achievement and learning motivation. J. Educ. Technol. Soc. 2020, 23, 1-15.

24. Goksu, I.; Islam Bolat, Y. Does the ARCS motivational model affect students' achievement and motivation? A meta-analysis. Rev. Educ. 2020, 1, 1-18. [CrossRef]

25. Fernández-Espínola, C.; Abad Robles, M.T.; Collado-Mateo, D.; Almagro, B.J.; Castillo Viera, E.; Giménez Fuentes-Guerra, F.J. Effects of cooperative-learning interventions on physical education students' intrinsic motivation: A systematic review and meta-analysis. IJERPH 2020, 17, 4451. [CrossRef]

26. Mailool, J.; Retnawati, H.; Rogahang, H.J.; Weol, W.; Waney, M.W. Synthesis and detection of publication bias in relationship between Motivation and teacher performance: A meta-analysis review. Univ. J. Educ. Res. 2020, 8, 6208-62162. [CrossRef]

27. Toste, J.R.; Didion, L.; Peng, P.; Filderman, M.J.; McClelland, A.M. A Meta-analytic review of the relations between motivation and reading achievement for K-12 students. Rev. Educ. Res. 2020, 90, 420-456. [CrossRef]

28. Dinçer, S. The effects of materials based on ARCS Model on motivation: A meta-analysis. EEO 2020, 19, 1016-1042. [CrossRef]

29. Koenka, A.C.; Linnenbrink-Garcia, L.; Moshontz, H.; Atkinson, K.M.; Sanchez, C.E.; Cooper, H. A meta-analysis on the impact of grades and comments on academic motivation and achievement: A case for written feedback. Educ. Psychol. 2019, 1-22. [CrossRef]

30. Kriegbaum, K.; Becker, N.; Spinath, B. The relative importance of intelligence and motivation as predictors of school achievement: A meta-analysis. Educ. Res. Rev. 2018, 25, 120-148. [CrossRef]

31. Volk, D.T. An Examination of the Relationship between School Climate, Self-Determined Academic Motivation, and Academic Outcomes among Middle and High School Students. Ph.D. Thesis, University of Connecticut, Storrs, CT, USA, 2020 ; p. 2435.

32. Murthy, S.A.P.; Jiar, Y.K. A meta-analysis of the relationships among students' anxiety, motivation and attitudes in learning English as Second Language. Man India 2017, 97, 349-361.

33. Higgins, K.; Huscroft-D' Angelo, J.; Crawford, L. Effects of technology in mathematics on achievement, motivation, and attitude: A meta-analysis. JCER 2019, 57, 283-319. [CrossRef]

34. Ergen, B.; Kanadli, S. The effect of self-regulated learning strategies on academic achievement: A meta-analysis study. Eurasian J. Educ. Res. 2017, 17, 55-74. [CrossRef]

35. Lazowski, R.A.; Hulleman, C.S. Motivation interventions in education: A meta-analytic review. Rev. Educ. Res. 2016, 86, 602-640. [CrossRef]

36. Hagger, M.S.; Chatzisarantis, N.L. The trans-contextual model of autonomous motivation in education: Conceptual and empirical issues and meta-analysis. Rev. Educ. Res. 2016, 86, 360-407. [CrossRef]

37. Bak, B.G. Comparison of academic motivation between gifted and non-gifted students: A meta-analysis. Korean J. Educ. Psychol. 2016, 30, 315-344. [CrossRef]

38. Maeng, U. L2 learning motivation and its correlates: A meta-analysis. Stud. Eng. Educ. 2016, 21, 1-37. [CrossRef]

39. Rios, J.A.; Ihlenfeldt, S.D.; Chavez, C. Are accommodations for english learners on state accountability assessments evidencebased? A multistudy systematic review and meta-analysis. Educ. Meas. Issues Pract. 2020, 39, 65-75. [CrossRef]

40. Li, H.; Xiong, Y.; Hunter, C.V.; Guo, X.; Tywoniw, R. Does peer assessment promote student learning? A meta-analysis. Assess. Eval. High. Educ. 2020, 45, 193-211. [CrossRef]

41. Lee, H.; Chung, H.Q.; Zhang, Y.; Abedi, J.; Warschauer, M. The effectiveness and features of formative assessment in us k-12 education: A systematic review. Appl. Meas. Educ. 2020, 33, 124-140. [CrossRef]

42. Double, K.S.; McGrane, J.A.; Hopfenbeck, T.N. The impact of peer assessment on academic performance: A meta-analysis of control group studies. Educ. Psychol. Rev. 2020, 32, 481-509. [CrossRef]

43. Mortaz, H.S.; Jalili, M.; Masoomi, R.; Shirazi, M.; Nedjat, S.; Norcini, J. The utility of mini-Clinical Evaluation Exercise in undergraduate and postgraduate medical education: A BEME review: BEME Guide No. 59. Med. Teach. 2020, 42, 125-142. [CrossRef]

44. Hanshaw, S.L.; Dickerson, S.S. High fidelity simulation evaluation studies in nursing education: A review of the literature. Nurse Educ. Pract. 2020, 46, 1-9. [CrossRef]

45. Lai, J.W.; Bower, M. Evaluation of technology use in education: Findings from a critical analysis of systematic literature reviews. JCAL 2020, 36, 241-259. [CrossRef]

46. Zhao, J.; Xu, X.; Jiang, H.; Ding, Y. The effectiveness of virtual reality-based technology on anatomy teaching: A meta-analysis of randomized controlled studies. BMC Med. Educ. 2020, 10, 1-10. [CrossRef]

47. Lester, A.M.; Chow, J.C.; Melton, T.N. Quality is critical for meaningful synthesis of afterschool program effects: A systematic review and meta-analysis. J. Youth Adolesc. 2020, 49, 369-382. [CrossRef] [PubMed]

48. Reyes, D.L.; Dinh, J.; Lacerenza, C.N.; Marlow, S.L.; Joseph, D.L.; Salas, E. The state of higher education leadership development program evaluation: A meta-analysis, critical review, and recommendations. Leadersh. Q. 2019, 30, 1-15. [CrossRef]

49. Bryfonski, L.; McKay, T.H. TBLT implementation and evaluation: A meta-analysis. Lang. Teach. Res. 2019, 23, 603-632. [CrossRef]

50. Huisman, B.; Saab, N.; Van den Broek, P.; Van Driel, J. The impact of formative peer feedback on higher education students' academic writing: A Meta-Analysis. Assess. Eval. High. Educ. 2019, 44, 863-880. [CrossRef]

51. Gegenfurtner, A.; Ebner, C. Webinars in higher education and professional training: A meta-analysis and systematic review of randomized controlled trials. Educ. Res. Rev. 2019, 28, 1-19. [CrossRef] 
52. Hurwitz, L.B. Getting a read on ready to learn media: A meta-analytic review of effects on literacy. Child Dev. 2019, 90, 1754-1771. [CrossRef]

53. Castro, M.D.B.; Tumibay, G.M. A literature review: Efficacy of online learning courses for higher education institution using meta-analysis. Educ. Inf. Technol. 2019, 1, 1-19. [CrossRef]

54. Petersen, J. Gender difference in verbal performance: A meta-analysis of United States state performance assessments. Educ. Psychol. Rev. 2018, 30, 1269-1281. [CrossRef]

55. Maan, J.; Hussain, I.; Sharma, K. Curriculum assessment of teacher education program in physical education: A meta-analysis. Int. J. Educ. Sci. 2018, 22, 37-44. [CrossRef]

56. Hamad, R.; Elser, H.; Tran, D.C.; Rehkopf, D.H.; Goodman, S.N. How and why studies disagree about the effects of education on health: A systematic review and meta-analysis of studies of compulsory schooling laws. Soc. Sci. Med. 2018, 212, 168-178. [CrossRef] [PubMed]

57. Cui, C.; Li, Y.; Geng, D.; Zhang, H.; Jin, C. The effectiveness of evidence-based nursing on development of nursing students' critical thinking: A meta-analysis. Nurse Educ. Today 2018, 65, 46-53. [CrossRef] [PubMed]

58. Panadero, E.; Jonsson, A.; Botella, J. Effects of self-assessment on self-regulated learning and self-efficacy: Four meta-analyses. Rev. Educ. Res. 2017, 22, 74-98. [CrossRef]

59. Conn, K.M. Identifying effective education interventions in sub-Saharan Africa: A meta-analysis of impact evaluations. Rev. Educ. Res. 2017, 87, 863-898. [CrossRef]

60. Vo, H.M.; Zhu, C.; Diep, N.A. The effect of blended learning on student performance at course-level in higher education: A meta-analysis. Stud. Educ. Eval. 2017, 53, 17-28. [CrossRef]

61. Yue, M.; Zhang, M.; Zhang, C.; Jin, C. The effectiveness of concept mapping on development of critical thinking in nursing education: A systematic review and meta-analysis. Nurse Educ. Today 2017, 52, 87-94. [CrossRef] [PubMed]

62. Li, H.; Xiong, Y.; Zang, X.L.; Kornhaber, M.; Lyu, Y.; Chung, K.S.; Suen, H.K. Peer assessment in the digital age: A meta-analysis comparing peer and teacher ratings. Assess. Eval. High. Educ. 2016, 41, 245-264. [CrossRef]

63. Sung, Y.T.; Chang, K.E.; Liu, T.C. The effects of integrating mobile devices with teaching and learning on students' learning performance: A meta-analysis and research synthesis. Comput. Educ. 2016, 94, 252-275. [CrossRef]

64. Zhou, J.; Zhou, S.; Huang, C.; Xu, R.; Zhang, Z.; Zeng, S.; Qian, G. Effectiveness of problem-based learning in Chinese pharmacy education: A meta-analysis. BMC Med. Educ. 2016, 16, 1-12. [CrossRef] 